\title{
Stress Measurement Using Magnetic Barkhausen Noise and Metal Magnetic Memory Testing
}

\author{
Ping WANG ${ }^{1}$, Shougao ZHU ${ }^{1}$, Gui Yun TIAN ${ }^{1,2}$, Haitao WANG ${ }^{1}$, Xin WANG ${ }^{1}$ \\ ${ }^{1}$ School of Automation, Nanjing University of Aeronautics and Astronautics, 29 Yudao St., \\ Nanjing 210016, China; Email: zeit@ 263.net \\ ${ }^{2}$ School of Electrical, Electronic and Computer Engineering, Merz Court, University of \\ Newcastle Upon Tyne, NE1 7RU, Newcastle Upon Tyne, UK
}

\begin{abstract}
This paper describes a new compound magnetic testing system using magnetic Barkhausen Noise (BN) and Metal Magnetic Memory (MMM) testing technique for stress measurement. BN has become an important nondestructive technique due to its exceptional material and stress characterization capabilities. MMM is recently developed technique with special ability for defect detection, stress detection and stress history record capacity. In the applied tensile experiment, BN and MMM signal were acquired via BN measurement system and EMS-2003 MMM instrument. Relationships between magnetic signals and applied tensile stresses were derived from experiment results. The correlation of the two methods was investigated. Conclusions were derived based on the experiment results. Further research directions were also highlighted.
\end{abstract}

Keywords: Magnetic Barkhausen Noise; Metal Magnetic Memory; Stress

\section{Introduction}

Ferromagnetic materials have been widely used in many industry fields such as machining, petroleum, railway traffic and aviation due to good elasticity and plasticity and many other good qualities, such as unyielding and enduring, etc. But breakdown of the products made of ferromagnetic materials sometimes may occur which can cause serious problems and may endanger people's life. Stress concentration is the main reason to cause fatigue failure and damage $^{[1]}$. However the early damage, especially the hidden that with no-continuance in it is very difficult to be taken effective evaluation.

Among several non-destructive techniques used for material evaluation ${ }^{[2-5]}$, such as X-ray, blind hole drilling, eddy current, $\mathrm{BN}^{[6,7]}$ offers exceptional material and stress characterization capabilities, good reliability, and high inspection speed, but the capacity for the detection of 
geometrical discontinuities is relatively poor, while $\mathrm{MMM}^{[8,9]}$ offer good defect detection, stress detection and stress history record capacity.

Table 1. Capabilities of selected NDT methods for stress inspection

\begin{tabular}{|c|c|c|c|c|c|}
\hline $\begin{array}{c}\text { Inspection } \\
\text { technique }\end{array}$ & $\begin{array}{c}\text { Spatial } \\
\text { resolution }\end{array}$ & Penetration & $\begin{array}{c}\text { Defect } \\
\text { detection }\end{array}$ & $\begin{array}{c}\text { Stress } \\
\text { detection }\end{array}$ & $\begin{array}{c}\text { Stress } \\
\text { history }\end{array}$ \\
\hline $\begin{array}{c}\text { Barkhausen } \\
\text { Noise }\end{array}$ & Fair & $0.01-1 \mathrm{~mm}$ & Poor & Good & No \\
\hline $\begin{array}{c}\text { Metal Magnetic } \\
\text { Memory }\end{array}$ & Fair & $\begin{array}{c}\text { Senesitivity decreases } \\
\text { with depth }\end{array}$ & Good & Good & Good \\
\hline
\end{tabular}

In this paper, BN and MMM testing technique were adopted for applied tensile stress measurement. Relationships of $\mathrm{BN}$ and MMM signal to stress were drawn from the tensile experiments. The correlation of the two methods was investigated. The paper finishes with the conclusions and an outline of further work.

\section{Background and theory}

In 1919, Barkhausen found that magnetization changes in a piece of iron induced a voltage in a pick-up coil which produced "noise" in an earphone, which was called "Barkhausen noise". Incidentally, this experiment gave the first evidence of the presence of magnetic domains in a ferromagnetic material. $\mathrm{BN}$ is detected as a series of voltage pulses appearing on the extension of a coil placed near or involving a ferromagnetic sample subjected to a varying external field. Researches for years have shown that $\mathrm{BN}$ results from the reversible and irreversible displacement of $180^{\circ}$ and non- $180^{\circ}$ domain walls, or by abrupt rotation of domain magnetization vectors at higher magnetic fields ${ }^{[10]}$. BN then gradually developed into a new non-destructive testing technique, namely Barkhausen Noise method. In recent years it has been applied for on-line inspection in manufacturing industries such as automotive, aerospace and metallurgical machinery.

MMM produced by researchers in Russia in the late 1990s, which is based on the magneto-mechanical effect, can estimate the early damage degree of ferromagnetic materials using MMM signals effect occurred under both of applied load and earth magnetic field existing. Not only stress concentration zones but also defects in the ferromagnetic materials can be found by MMM, so it has been used widely in the engineering field due to simple operation. The basic principle of $\mathrm{MMM}^{[11]}$ can be expressed as: due to the self magnetization of ferromagnetic engineering structures by ambient fields, $\operatorname{Hp}(\mathrm{x})$ exhibits a peak in field strength and $\mathrm{Hp}(\mathrm{y})$ changes its polarity around stress concentration zones or macroscopic defects, where $\mathrm{Hp}(\mathrm{x})$ is magnetic flux leakage component parallel to the material surface and $\mathrm{Hp}(\mathrm{y})$ is that perpendicular to the material surface.

\section{Experiment setup and typical BN signal}


BN measurement system is schematically shown in Fig.1. A signal generator CALTEK CA1640-02 supplies a sinusoidal wave of $10 \mathrm{~Hz}$, which is amplified by a power amplifier Newtons4th LPA05B that feeds a 400 turn excitation coil in order to produce magnetic saturation in the samples. BN signal from GMR sensor AAH002-02 was then acquired to PC via an Adlink 2010 4-channel data acquisition card. MMM signal was acquired by a MMM diagnostics instrument (EMS-2003). Data processing was performed in Matlab.

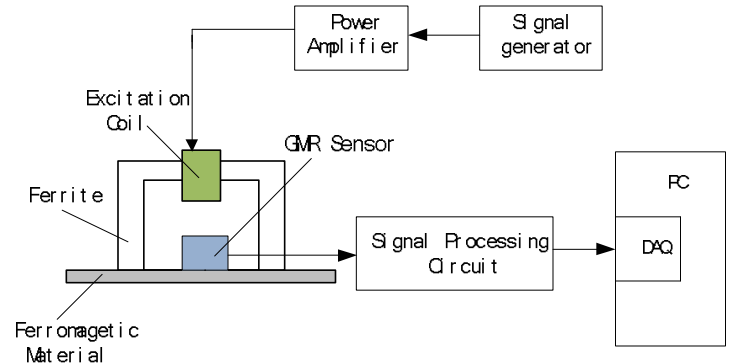

(a)

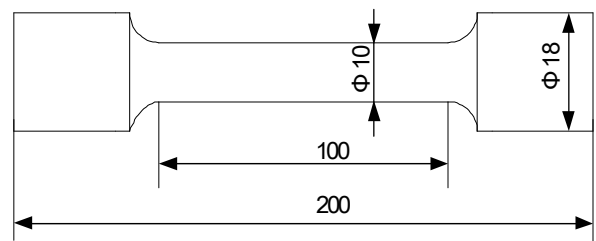

(b)

Figure 1. BN measurement system (a) and tensile sample dimensions with size in $\mathrm{mm}$ (b)

For all the experimental measurements, the used samples were made of low carbon steel. And figure $1 \mathrm{~b}$ and table 1 gives dimensions and mechanical properties of the sample respectively.

Table 1. Mechanical properties of low carbon steel sample

\begin{tabular}{|c|c|c|c|}
\hline$\sigma_{\mathrm{s}} / \mathrm{Mpa}$ & $\sigma_{\mathrm{b}} / \mathrm{Mpa}$ & $\mathrm{E} / \mathrm{Gpa}$ & $\mu$ \\
\hline 275.6 & 421.8 & 216 & 0.28 \\
\hline
\end{tabular}

A material testing machine CSS-44100 was used to apply tensile force from $0 \mathrm{kN}$ to $30 \mathrm{kN}$ with a loading velocity of $4 \mathrm{~mm} / \mathrm{min}$.

Figure 2a shows a typical filtered BN signal from GMR sensor in time domain and figure $2 \mathrm{~b}$ gives BN signal in frequency domain. It can be seen from the plot that the greatest amplitude of $\mathrm{BN}$ signal comes from the excitation signal is exhibiting its greatest rate of change, and it has a wide bandwidth, about $1 \mathrm{kHz}-500 \mathrm{kHz}$.

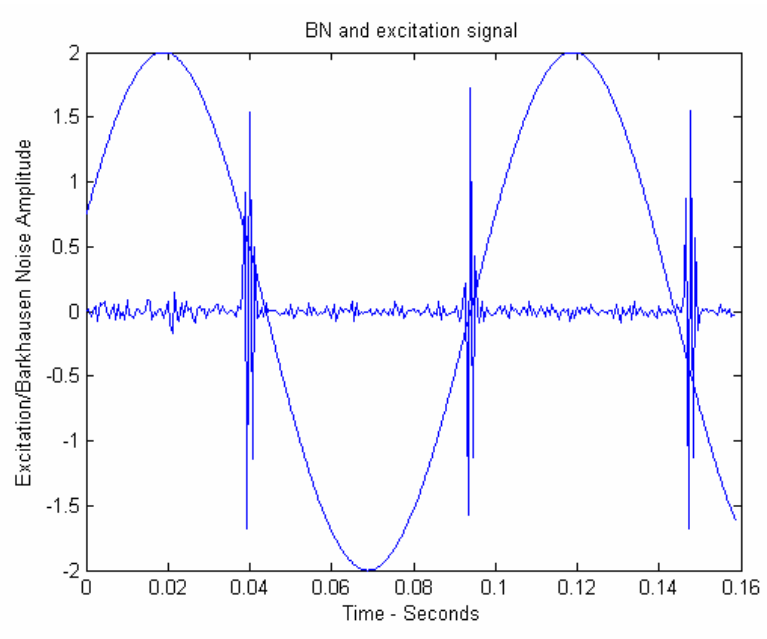

(a)

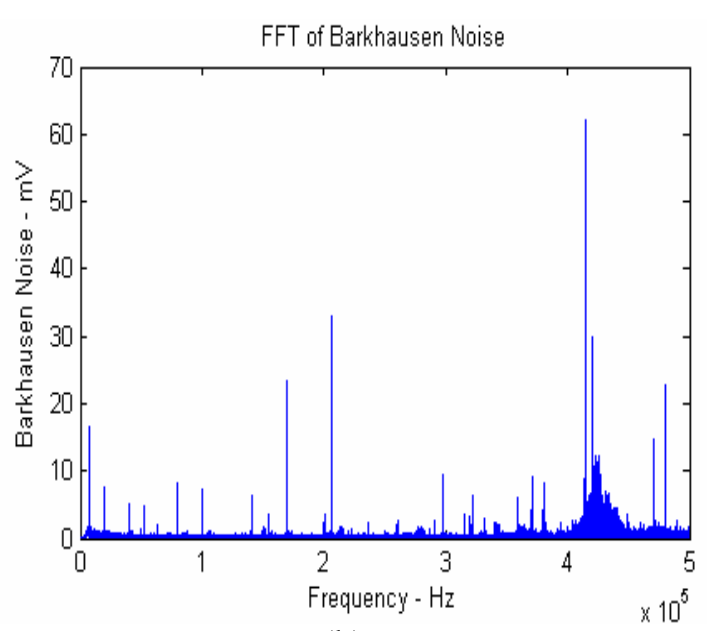

(b) 
Figure 2. Excitation signal and signal from GMR sensor filtered to leave Barkhausen noise (a) and FFT of Barkhausen noise (b)

As the signal is quite complicated, RMS value of processing signal was adopted for analysis, which is given by:

$$
\psi_{\mathrm{X}}=\sqrt{\frac{1}{n} \sum_{i=0}^{n-1} X_{i}^{2}}
$$

\section{Active stress measurement}

\subsection{BN for active stress measurement}

Figure 3 shows after an increase the $\mathrm{BN}$ signal decreases with the increase of the applied stress. In the elastic stage, BN signal increases abruptly, and at the beginning of plastic stage $\mathrm{BN}$ drops quickly then slowly. BN signal is quite sensitive to stress during the elastic stage, and has a good linear relationship with stress in the plastic stage.

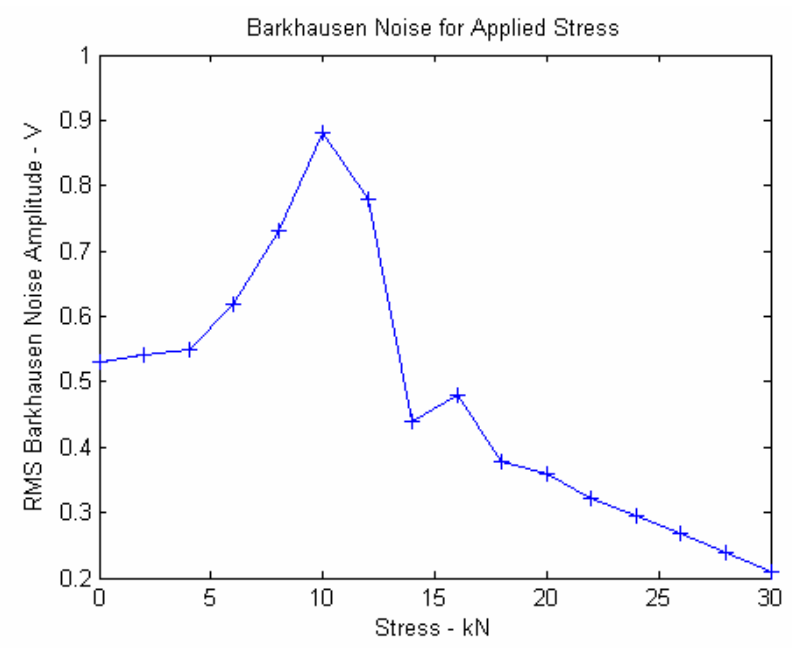

Figure 3. RMS Barkhausen noise amplitude for applied stress

In the elastic deformation region, due to the theory of magneto-elastic energy ${ }^{[12]}$, with the applied tensile stress, more domain walls shall emerge in the stress direction. Since BN is associated with the pinning of these domain walls as they move during magnetization, the BN signal increases in the direction of tensile stress, which is illustrated in Figure 3.

Once the plastic region is reached, however, the macroscopic elastic strain remains approximately constant, with plastic deformation occurring through slip processes. These processes lead to a number of changes that potentially can alter magnetic behavior ${ }^{[12]}$ :

1. An increase in the number of microscopic pinning sites in the form of dislocation tangles and cell boundaries, which act as obstacles to domain wall movement.

2. Development of a crystallographic texture, which may alter the magnetic easy axis, direction, and finally.

With the increase of stress, the influence of substructure obstacles the pinning sites to domain 
wall motion, and the development of a crystallographic texture cause the decrease of domains. With reasons above, $\mathrm{BN}$ emission decreases in addition to the change of applied tensile stress.

\subsection{Active stress measurement using MMM}

In the experiment, the $\mathrm{Hp}(\mathrm{y})$ value and $\mathrm{Hp}(\mathrm{y})$ gradient $\mathrm{dHp}(\mathrm{y}) / \mathrm{dx}$ of the middle point of the sample were measured via MMM testing instrument EMS-2003. Figure 4a gives Hp(y) value for applied tensile stress. From the plot, it is very clear that, $\operatorname{Hp}(\mathrm{y})$ value is around zero with the applied tensile stress. From the theory of MMM, it is thought that the middle point of the tensile sample is around the stress concentration zone. However, no further variation rule is observed from the $\mathrm{Hp}(\mathrm{y})$ - Stress curve.

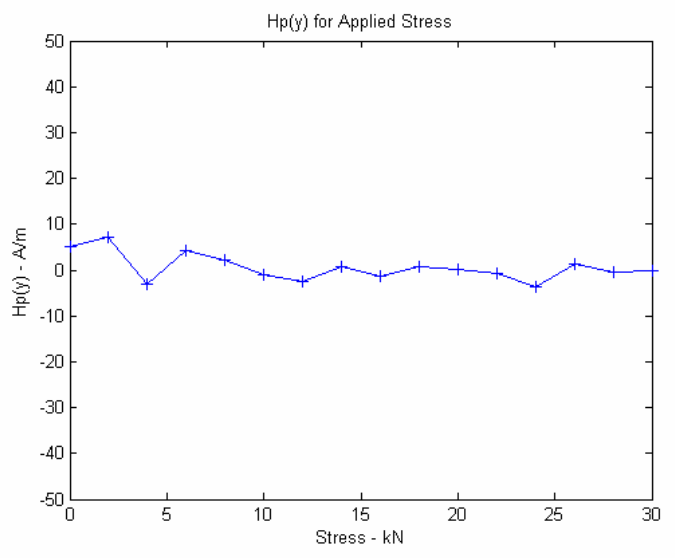

(a)

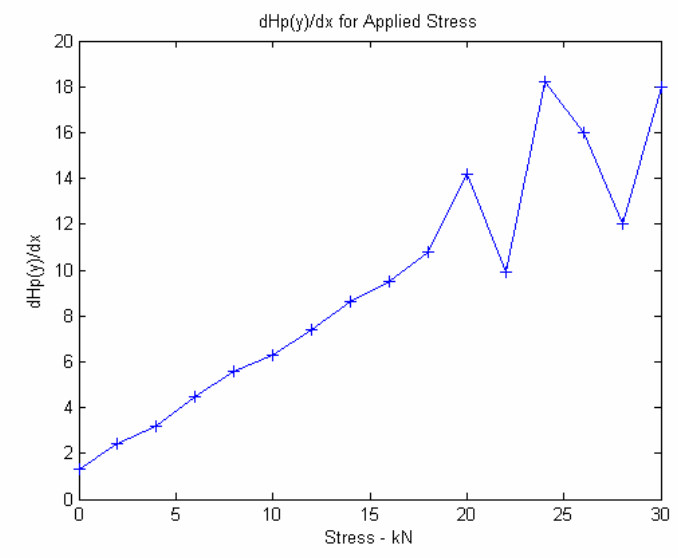

(b)

Figure 4. $\mathrm{Hp}(\mathrm{y})$ for applied tensile stress (a) and $\mathrm{dHp}(\mathrm{y}) / \mathrm{dx}$ for applied tensile stress (b)

From figure $4 \mathrm{~b}, \mathrm{dHp}(\mathrm{y}) / \mathrm{dx}$ value has a good linear relationship with applied tensile stress. However, $\mathrm{dHp}(\mathrm{y}) / \mathrm{dx}$ value exhibits arbitrary change with the applied stress.

Same as the mechanism that has been discussed above about BN signal, in the elastic stage, under the applied load and earth magnetic field existing, the rotation of magnetic domains dominates the increase of $\mathrm{Hp}(\mathrm{y})$ absolute value of the points around the middle point, thus $\mathrm{dHp}(\mathrm{y}) / \mathrm{dx}$ increases. When the plastic stage is reached, due to lattice dislocations and micro-defects and eventually macro-defects, causes zero drift of $\mathrm{Hp}(\mathrm{y})$ value thus arbitrary change of $\mathrm{dHp}(\mathrm{y}) / \mathrm{dx}$ value ${ }^{[13]}$.

\section{Conclusions}

From the experiments results above, we know that BN and MMM share the same mechanism, one difference lies between them is that $\mathrm{BN}$ relies on the time-varying magnetic field generated by excitation coil, while MMM relies on the earth magnetic field. I.e., BN is due to external magnetization and MMM is due to "self magnetization". And due to the good linear relationship with applied tensile stress at different stages, the combination of the two techniques offers the potential for complementary data acquisition in the measurement system. Also, due to good defect detection ability of MMM, the characterization of stresses and defects could be achieved 
via data fusion of the two techniques.

\section{References}

[1] M.N. James, D.J. Hughes, Z. Chen, H. Lombard, D.G. Hattingh and D. Asquith et al, Residual stresses and fatigue performance [J]. Engineering Failure Analysis, 2007, 14 (2), pp. 384-395.

[2] V.S. Pisarev, V.V. Balalov, V.S. Aistov, M.M. Bondarenko and M.G. Yustus, Reflection hologram interferometry combined with hole drilling technique as an effective tool for residual stresses fields investigation in thin-walled structures [J]. Opt Lasers Eng 366 (2001), pp. 551-597.

[3] Takayuki MUROTANI, Tomonori YANO, Hajime HIROSE and Akira IKENAGA, Applications of X-ray stress measurement for interface area of $\mathrm{Ni3Al}$ system intermetallic compound coating [J]. International Centre for Diffraction Data, 2004, Advances in X-ray Analysis, Volume 47, pp. 385-389.

[4] John. W. Wilson, Gui Yun Tian, Pulsed electromagnetic methods for defect detection and characterization [J]. NDT\&E International, 2007, 40, pp. 275-283.

[5] G. Y. Tian, A. Sophian, D. Taylor, J Rudlin, Wavelet-based PCA Defect Classification and Quantification for Pulsed Eddy Current NDT [J]. IEE Proc. Science, Measurement \& Technology, 152( 4), July 2005, pp. 141-148.

[6] A. Dhar and D.L. Atherton, Effects of magnetic flux density and tensile stress on the magnetic Barkhausen noise in pipeline steel [J]. Non-Destruct Test Eval 10 (1993), pp. 287-294.

[7] T.W. Krause, L. Clapham, A. Pattantyus and D.L. Atherton, Investigation of the stress-dependent magnetic easy axis in steel using magnetic Barkhausen noise [J]. Appl Phys 798 (1996), pp. 4242-4252.

[8] Doubov Anatoli A. Diagnostics of Metal Items and Equipment by Means of Metal Magnetic Memory [A]. Proc of CHSNDT 7th Conference on NDT and International Research Symposium [C]. Shantou China: Non-Destructive Testing Institution, CEMS, 1999, pp. 181-187.

[9] John W. Wilson, Gui Yun Tian and Simon Barrans. Residual magnetic field sensing for stress measurement [J]. Sensors and Actuators A: Physical. In Press, Corrected Proof, Available online 20 September 2006.

[10] L. Clapham, S. White, J. Lee and D.L. Atherton, Magnetic easy axis development in steel—the influence of manufacturing [J]. Appl Phys 88 (2000) (4), pp. 2163-2165.

[11] A. Dubov. Principal features of metal magnetic memory method and inspection tools as compared to known magnetic ndt methods [A]. Proceedings of the 16th Annual World Conference on Non-Destructive Testing [C]. Montreal, Canada, 30 August-3 September, 2004.

[12] C. G. Stefanita, D. L. Atherton and L. Clapham, Plastic versus elastic deformation effects on magnetic Barkhausen noise in steel [J]. Acta Materialia, Volume 48, Issue 13, 1 August 2000, Pages 3545-3551.

[13] Zhifang Liang, Wushen Li, Yingna, Wang Shiwu Bai, and Zhenkui Xue, Zero value character of metal magnetic memory signal [J]. Journal of Tianjin University, 39 (7), pp. 847-850. 